\title{
Spatial analysis of bluetongue cases and vaccination of Swiss cattle in 2008 and 2009
}

\author{
Katriina J. E. Willgert ${ }^{1}$, Birgit Schroedle ${ }^{2}$, Heinzpeter Schwermer ${ }^{3}$ \\ ${ }^{1}$ Royal Veterinary College, Royal College Street, London, NW1 OTU, United Kingdom; ${ }^{2}$ Biostatistics Division, \\ Institute of Social and Preventive Medicine, University of Zurich, CH-8001 Zurich, Switzerland; ${ }^{3}$ Monitoring \\ Unit, Federal Veterinary Office, Schwarzenburgstrasse 155, CH-3003 Bern, Switzerland
}

\begin{abstract}
Bluetongue (BT) is a vector-borne viral disease of ruminants. The infection is widespread globally with major implications for international animal trade and production. In 2006, BT virus serotype 8 (BTV-8) was encountered in Europe for the first time, causing extensive production losses and death in susceptible livestock. Following the appearance of BTV8 in Switzerland in 2007, a compulsory vaccination programme was launched in the subsequent year. Due to social factors and difficulties to reach animals on high pasture, the regional vaccination coverage varied across the country in both 2008 and 2009. In this study, the effect of vaccination on the spatial occurrence of BTV-8 and the associated relative disease risk in Switzerland in 2008 and 2009 were investigated by a spatial Bayesian hierarchical approach. Bayesian posterior distributions were obtained by integrated nested Laplace approximations, a promising alternative to commonly used Markov chain Monte Carlo methods. The number of observed BTV-8 outbreaks in Switzerland decreased notably from 2008 to 2009. However, only a non-significant association between vaccination coverage and the probability of a spatial unit being infected with BTV-8 was identified using the model developed for this study. The relative disease risk varied significantly across the country, with a higher relative risk of BTV-8 infection in western and north-western Switzerland where environmental conditions are more suitable for vector presence and viral transmission. Examination of the spatial correlation between disease occurrence, control measures and associated ecological factors can be valuable in the evaluation and development of disease control programmes, allowing prioritisation of areas with a high relative risk of disease.
\end{abstract}

Keywords: Bayesian hierarchical model, bluetongue, disease mapping, integrated nested Laplace approximation, vaccination, Switzerland.

\section{Introduction}

Bluetongue $(\mathrm{BT})$ is a non-contagious, arboviral disease of ruminants. The disease causes extensive economic losses in the livestock production sector and is listed as a notifiable disease by the World Organisation for Animal Health (OIE) (Mellor and Wittmann, 2002; Mintiens et al., 2008; Saegerman et al., 2008). The mortality rate can be as high as $50-70 \%$ in certain sheep breeds (Sellers, 1984; Elbers et al., 2008b) and infected countries are imposed with trade restrictions (Méroc et al., 2008). The aetiological agent of BT, bluetongue virus (BTV), belongs to the family Reoviridae and the genus Orbivirus (Mellor and Wittmann, 2002; Purse et al., 2005). At the present time, there are 24 recognised serotypes of BTV and a putative $25^{\text {th }}$ serotype has been identified in goats

Corresponding author:

Heinzpeter Schwermer

Monitoring Unit, Federal Veterinary Office

Schwarzenburgstrasse 155, CH-3003 Bern, Switzerland

Tel. +4131323 3053; Fax +41313239543

E-mail: heinzpeter.schwermer@bvet.admin.ch
(Hofmann et al., 2008; Saegerman et al., 2008). The clinical implications of BTV infection can range from asymptomatic to severe or even death. BT infection tends to be more pronounced in sheep than other domestic ruminants, such as cattle, where the disease often is sub-clinical (Sellers, 1984; Elbers et al., 2008a; Vellema, 2008).

The geographical distribution of $\mathrm{BT}$ is restricted to areas where there are susceptible animal hosts, competent vector species and suitable climatic conditions for viral transmission (Mellor et al., 2000; Mellor and Wittmann, 2002; Saegerman et al., 2008). BT is transmitted by biting midges of the genus Culicoides (Mellor and Wittmann, 2002; Purse et al., 2005). Climatic and environmental conditions, such as temperature, humidity and access to breeding sites limit the vector distribution (Sellers, 1984; Purse et al., 2005; Saegerman et al., 2008). In addition, BTV replication in the vector and host is temperature dependent and, although the vector can be active at temperatures above $10{ }^{\circ} \mathrm{C}$, viral replication in the vector becomes prominent at temperatures above 15$18{ }^{\circ} \mathrm{C}$ (Mellor, 2000; Wittmann et al., 2002; EFSA, 2007). 
In August 2006, BTV-8 emerged in the Netherlands, a serotype of BTV that had previously not been recorded in Europe (Elbers et al., 2008a; Mintiens et al., 2008). The infection spread rapidly across Europe in 2007 and 2008. Thus, when commercial inactivated vaccines against BTV-8 became available in 2008, several affected countries launched national vaccination campaigns in order to control the disease (Gethmann et al., 2009; Roy et al., 2009). Following the outbreak of BTV-8 in northern Europe in 2006, BT surveillance in Switzerland was intensified and increased disease awareness was encouraged among livestock keepers (Kluiters et al., 2008). For surveillance purposes, Switzerland was divided into 16 regions, hereafter referred to as BT-regions, based on animal population numbers, land area and administrative boundaries. The Principality of Liechtenstein composed a 17th BT-region (Figure 1; Kluiters et al., 2008). The first case of BTV-8 in Switzerland was detected in October 2007 (Hofmann et al., 2008). Switzerland initiated a vaccination campaign against BTV-8 in June 2008 with mandatory vaccination of cattle, sheep and goats (FVO, 2009). The vaccination campaign continued in 2009 with compulsory vaccination of cattle and sheep above 3 months of age (FVO, 2009). The vaccination programme was implemented locally by the cantonal veterinary offices and, consequently, the vaccination coverage varied regionally in both years, ranging from $24 \%$ to $82 \%$.

The effect of vaccination on the disease prevalence in a population depends on the achieved vaccination coverage, characteristics of the disease and attributes of the targeted group (Anderson and May, 1982).
Simulation models show that both the incidence and spatial spread of BTV-8 decrease with increasing vaccination coverage (Szmaragd et al., 2010a,b). By using adequate spatial models, the spatial association between disease management measures or ecological factors and disease prevalence can be explored. We applied a spatial Bayesian hierarchical approach to examine the spatial heterogeneity in relative disease risk and to investigate the association between BTV-8 occurrence and vaccination coverage in Switzerland in 2008 and 2009, while controlling for spatial variation in surveillance intensity. Since vaccination of susceptible animals reduces virus transmission and the probability of an animal acquiring infection (Szmaragd et al., 2010b), a negative correlation between vaccination coverage and the probability of a unit being infected with BTV-8 in a spatially defined area was expected.

\section{Materials and methods}

\section{Study area and study population}

Switzerland is a federal state, consisting of 20 cantons and six half cantons. The country covers an area of $41,285 \mathrm{~km}^{2}$ and is located north of latitude $46^{\circ} \mathrm{N}$ in central Europe. In the south, Switzerland is divided by the Alps with a maximum altitude of $4,700 \mathrm{~m}$ above sea level. Out of the total land area, $37 \%$ is used for agricultural purposes. Family driven farming enterprises are most predominant, with milk being the main product of livestock keeping.

The number of cattle farms and registered cattle on

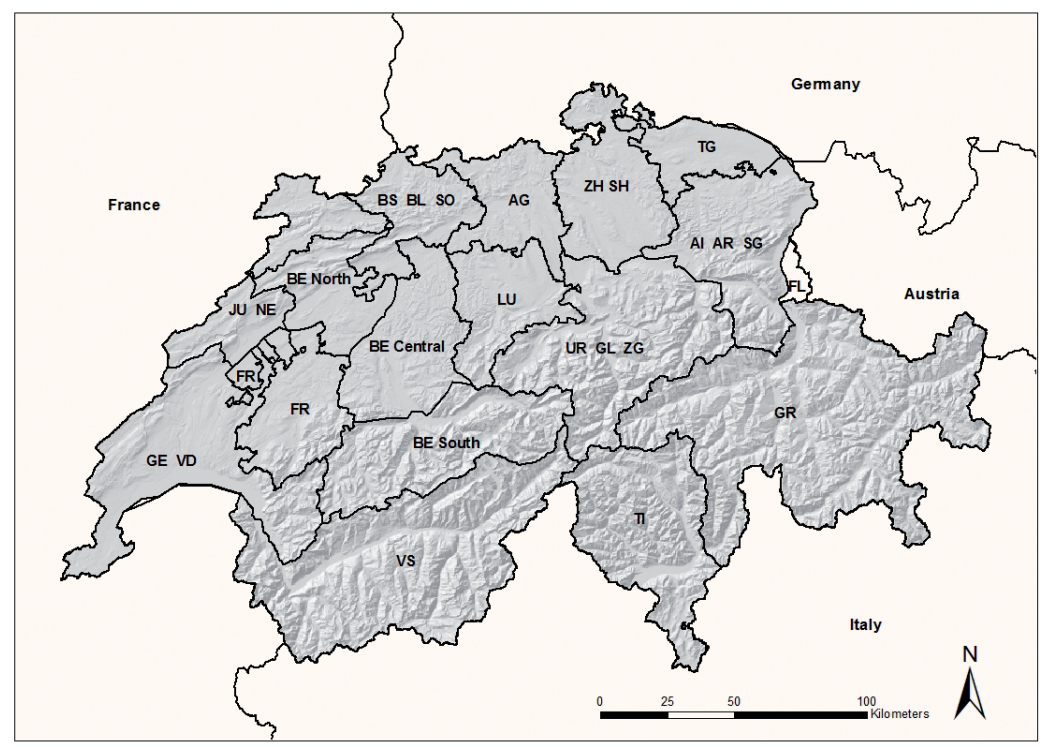

Fig. 1. Hillshade map of Switzerland showing the division of the Swiss cantons and Liechtenstein (FL) by their abbreviated names into 17 bluetongue regions. 
the $1^{\text {st }}$ of May in 2008 and 2009 were obtained from the Swiss Animal Movement Database (Tierverkehrsdatenbank TVD) and included in the study as the population at risk (2008: $\mathrm{n}=1,628,435$ animals/44,559 farms; 2009: $\mathrm{n}=1,642,613$ animals/44,202 farms). Since official disease control measures are applied locally by the cantonal veterinary authorities, animal and farm data were first considered at a regional level by aggregating animal data ("animal aggregated data") and farm data ("farm aggregated data”) for each BT-region. Secondly, pointreferenced data of each farm were analysed with explanatory variables specific to the location of the farm ("point-referenced data").

A total of 42,081 farms $(90.7 \%)$ were given Cartesian coordinates based on their TVD identification number. If no geographical coordinates were available, farms were assigned the centroid coordinates of their community. ArcGIS version 9.2 (ESRI; Redlands, CA, USA) was used to map and manage spatial information. Geographical coordinates of farms were referenced in the Swiss reference system $\mathrm{CH} 1903$. For the regional analysis, the geographical coordinates of each farm were spatially joined to a shapefile of the 17 BT-regions in order to estimate the population at risk per BT-region. In the analysis of point-referenced data, 4,323 farms (9.3\%) were excluded from the population at risk since they lacked geographical point coordinates. For farms included in the analysis of point-referenced data, the farm altitude ( $\mathrm{m}$ above sea level) was extracted from raster elevation map DHM25 (Federal Office of Topography swisstopo) with a spatial resolution of $5 \times 5 \mathrm{~m}$.

\section{Disease data}

BTV-8 case data of cattle from 2008 and 2009 were extracted from the Swiss database of notifiable diseases (InfoSM) maintained by the Federal Veterinary Office (FVO). Since different surveillance activities may target separate characteristics of the population and disease (Del Rio Vilas and Pfeiffer, 2009), data from three simultaneous but independent surveillance programmes were included in the analysis: reported cases of clinical suspicion, official targeted surveillance where 10 cattle from 20 randomly selected farms of each BT-region were sampled in February to March each year (FVO, 2009), and pre-movement testing. For pre-movement tested animals, the animal identification number was linked to the TVD database in order to identify the last farm location. Data were aggregated annually since the time delay in reporting disease following infection of an animal may vary. Moreover, the time of infection of sub-clinical cases detected through the official surveillance programme and pre-movement testing is unknown. Seeing that BTV infection generally is not detected in cattle herds until 2 months after the beginning of the vector active season (Bishop et al., 2004, Conraths et al., 2009), which in Switzerland was in April in both 2008 and 2009 (FVO, 2009), cases detected through the official surveillance programme or pre-movement testing before the $1^{\text {st }}$ of June were assumed to belong to the previous BT season.

An animal was considered BTV-8 positive if it had a positive polymerase chain reaction (PCR) result for BTV-8 from the national reference laboratory for BT, the Institute of Virology and Immunoprophylaxis (IVI; Mittelhäusern). A farm was regarded BTV-8 positive if at least one positive animal was detected at the farm. The diagnostic method was assumed to be $100 \%$ sensitive and specific.

\section{Vaccination data}

Yearly BTV-8 vaccination data of cattle were acquired from FVO. An animal was considered vaccinated against BTV-8 following two vaccine doses administrated within an interval of 21 to 63 days. A farm was regarded vaccinated if at least one animal at that farm had been vaccinated. Vaccine efficacy was assumed to be $100 \%$. Any delays in reaching full protection were neglected.

\section{Spatial modelling}

\section{Analysis of regionally aggregated data.}

To estimate the relative risk of one unit being infected at a regional level, a spatial and spatio-temporal disease mapping model constructed in a Bayesian hierarchical framework was developed. Three hierarchical stages were specified as explained by Clayton and Bernardinelli (1992). The first stage describes the observed data, in this case the number of infected units per BT-region, as a function of the region-specific relative risk of disease. At stage two, the relative risk of disease for each region is modelled. The hyperpriors for all variance parameters of stage two are specified at stage three. It was assumed that the number of infected units $y_{i}$ in a region $i(i=1, \ldots, 17)$ has a Poisson distribution:

$$
y_{i} \sim \text { Poisson }\left(\lambda_{i} \cdot n_{i}\right)
$$


where $\lambda_{i}$ is the probability of one unit, either animal or farm, being BTV-8 infected in region $i$, and $n_{i}$ the population at risk in each BT-region. The relative risk parameter $\lambda_{i}$, described by Besag et al. (1991), is specified using a linear predictor:

$$
\eta_{i}=\log \left(\lambda_{i}\right)=\mu+v_{i}+\psi_{i}
$$

where $\mu$ is the intercept. The regional term $v_{i}$ and global term $\psi_{i}$ account for any unmeasured explanatory variables considered in the model (Clayton et al., 1993). The term $v_{i}$ is a spatially unstructured variable that accounts for unmeasured risk factors that vary between areas, whereas $\psi_{i}$ is assumed to be structured in space and models the effect of the location, accounting for the assumption that geographically close areas are more related than distant areas (Clayton et al., 1993; Durr et al., 2005). Hence, the regional term $v_{i}$ was modelled as an independent identically (i.i.d.) normal distributed random effect, while the global term $\psi_{i}$ was modelled as a so-called intrinsic Gaussian Markov random field (IGMRF) of first order on an irregular lattice (Rue and Held, 2005). Such an IGMRF accounts for spatial autocorrelation by assuming that the conditional distribution of $\psi_{i}$ in region $i$ depends on the $\psi_{j}$ in neighbouring regions $j$. An adjacency matrix of the BT-regions was constructed, where neighbours of each region, defined by a shared common boundary, were counted and listed. To all variances, an inverse gamma hyper-prior IGa $(1.0,0.01)$ was assigned (Bernardinelli et al., 1995a). To account for any additional over-dispersion, the data could be modelled using a negative binomial distribution (Gschössl and Czado, 2008), which includes an additional dispersion parameter. However, the deviance information criterion (DIC) (Spiegelhalter, 2002) was higher for all models assuming a negative binomial distribution than for the corresponding Poisson models, indicating that a Poisson distribution was sufficient for this study.

Two region-specific explanatory covariates were included in the analysis to account for any spatial variation in relative disease risk: vaccination coverage $\left(\right.$ vacc_cov $\left._{i}\right)$, calculated as the number of units vaccinated divided by the total number of units, and surveillance intensity (surv_int ${ }_{i}$ ), estimated by dividing the number of tested units by the total number of units:

$$
\begin{aligned}
\eta_{i}= & \log \left(\lambda_{i}\right)=\mu+\beta_{1} \cdot \text { vacc_cov }_{i}+ \\
& +\beta_{2} \cdot \text { surv_int }_{i}+v_{i}+\psi_{i}
\end{aligned}
$$

where $\beta$ denotes a fixed linear effect of the explanatory variable. The surveillance intensity in each region was included to assure that any variation in estimated disease risk was not the product of regionally differing surveillance efforts. Since the cantonal veterinary services implement legislation of animal health at a local level (Rüsch and Kihm, 2003), the surveillance intensity varies regionally and reporting of suspected disease may be biased in space and time by varying disease awareness (Kluiters et al., 2008; Del Rio Vilas and Pfeiffer, 2009).

Due to the sparsity of BTV-8 positive cases in 2009, a spatial analysis was only conducted for 2008. To assess if there was any significant change in the probability of a unit being infected between 2008 and 2009, a spatio-temporal disease mapping model was adopted. A random intercept $\gamma_{t}$ for each year $t$ was added to formula (3) (Knorr-Held, 2000):

$$
\begin{aligned}
\eta_{i t}= & \log \left(\lambda_{i t}\right)=\mu+\beta_{1} \cdot \text { vacc_cov }_{i}+ \\
& +\beta_{2} \cdot \text { surv_int }_{i}+v_{i}+\psi_{i}+\gamma_{t}
\end{aligned}
$$

This is a version of the standard non-parametric space-time model introduced by Knorr-Held (2000). Since the data considered were restricted to 2 years, a random intercept for each time point was preferred to a linear trend (Bernardinelli et al., 1995b). Due to the sparseness of BTV-8 cases, especially in 2009, any interactions between space and time were neglected. An inverse gamma hyper-prior IGa $(1.0,0.01)$ was assigned to the variance of $\gamma_{t}$.

The four mountain BT-regions Bern south (BE South), Valais (VS), Ticino (TI) and Grisons (GR) (Fig. 1) were excluded from the analysis at regional level since the risk factors associated with BT are believed to differ considerably from the rest of the country in these areas due to the distinct environmental conditions observed in the Alps (Racloz et al., 2008). In the Alps, the microclimate varies within short distances, making it difficult to form a covariate that is valid for the whole region to adjust for the local environmental conditions.

\section{Analysis of point-referenced data.}

For the analysis of point-referenced farm data, a generalised Bayesian geoadditive model was adopted (Kneib and Fahrmeir, 2006; Musio et al., 2008), where the exact location of the unit in space is known. The location of each farm in terms of easting and northing was denoted as $s_{i j}$. Since farms can be either BTV-8 positive or negative, it was assumed that the response $y_{i j}$ followed a binomial distribution:

$$
y_{i j} \sim \operatorname{Binom}\left(\pi_{i j}, 1\right)
$$


where $\pi_{i j}$ denotes the probability of a farm at location $s_{i j}$ being infected, modelled by a latent Gaussian field of the form:

$$
\begin{gathered}
\eta_{i j}=\operatorname{logit}\left(\pi_{i j}\right)=\mu+\beta_{1} \cdot \text { vacc_cov }_{i j}+ \\
+\beta_{2} \cdot \text { surv_int }_{i j}+f\left(s_{i j}\right)
\end{gathered}
$$

where the vaccination coverage $\left(\right.$ vacc_cov $\left._{i j}\right)$ was given the value 1 if at least one animal at the farm had been vaccinated and otherwise zero. To account for varying surveillance efforts within the study area, a 0-1-index for the surveillance intensity (surv_int ${ }_{i j}$ ) was also included as an explanatory variable. The location effect $f$ of each farm was modelled as a Gaussian Markov random field on a two-dimensional lattice (Rue and Held, 2005). The easting and northing of each farm were assigned to a regular grid with $I$ rows and $J$ columns (Besag and Kooperberg, 1995), where $I=100$ and $J=80$ based on the shape of Switzerland. An intrinsic second order random walk prior was adopted (Rue and Held, 2005), where the full conditional mean of a node $(i, j)$ on the grid depends on the four direct neighbours on the grid, the four diagonal neighbours on the grid and the four direct neighbours of second order. Hence, a node $(i, j)$ borrows strength from its 12 closest spatial neighbours. The size of the residual spatial effect is controlled by the variance, $\sigma_{f}^{2}$. An inverse gamma hyper-prior IGa $(1.0,0.01)$ was assigned to $\sigma_{f}^{2}$.

Due to the scarcity of BTV- 8 cases, a linear spatial trend surface was also adapted with a linear predictor defined as:

$$
\begin{aligned}
\eta_{i j}= & \operatorname{logit}\left(\pi_{i j}\right)=\mu+\beta_{1} \cdot \text { vacc_cov }_{i j}+ \\
& +\beta_{2} \cdot \text { surv_int }_{i j}+\beta_{4} \cdot i+\beta_{5} \cdot j
\end{aligned}
$$

where the indexes $i$ and $j$ denote the location of a farm on the spatial lattice. The conditional mean of each farm depends on the average response of the farms in the same row and the same column. This formulation gives a more global east-west and north-south effect compared to the more localized, autoregressive two-dimensional second order random walk discussed previously.

In contrast to the aggregated data, the exact altitude (altitude ${ }_{i j}$ ) of each farm is known and can be put into model (7) as an indicator of temperature dependent dynamics of the vector population and viral replication:

$$
\begin{aligned}
\eta_{i j}=\operatorname{logit} & \left(\pi_{i j}\right)=\mu+\beta_{1} \cdot \operatorname{vacc}_{-} \operatorname{cov}_{\mathrm{ij}}+\beta_{2} \cdot \text { surv_int }_{\mathrm{ij}} \\
+ & \beta_{3} \cdot \text { altitude }_{\mathrm{ij}}+\beta_{4} \cdot i+\beta_{5} \cdot j
\end{aligned}
$$

Since this model is able to, at least partly, adjust for different environmental conditions in mountain areas, all data were used for this analysis.

\section{Model choice and computation}

Models were compared and selected using DIC, which takes into consideration the posterior mean deviance, a Bayesian measure of model fit, and the complexity of the model. A smaller DIC indicates a better fit of the model (Spiegelhalter, 2002).

All models were computed using integrated nested Laplace approximations (INLA), a recently proposed method for approximate Bayesian inference within latent Gaussian models. INLA has various applications, ranging from generalized mixed models to dynamic and spatio-temporal models (Rue et al., 2009; Schrödle and Held, 2010). Furthermore, INLA outperforms traditional Markov chain Monte Carlo (MCMC) methods in terms of computational time while providing very precise estimates (Rue et al., 2009). The analyses can be conducted within a powerful R environment ( $\mathrm{R}$ Development Core Team, 2010) using the R-INLA package available at www.rinla-org. All analyses in this study were run using the R-INLA version built on 22 October 2010.

\section{Results}

A total of 69 BTV-8 infected holdings and 131 BTV8 infected cattle were recorded in 2008. Three affected holdings with one infected animal each were documented in 2009 (Fig. 2). Summary statistics for the 17 BT-regions are displayed in Table 1.

\section{Parameter results of regional disease mapping models}

For all regional models, the posterior distribution of the fixed effect of vaccination had a negative posterior mean (Table 2), suggesting that increasing vaccination coverage reduced the probability of a unit being infected. However, all credible intervals contained the value zero, meaning that the model did not identify a significant association between the level of regional vaccination and the probability of a unit being infected.

The number of tested units from reported cases of clinical suspicion, official targeted surveillance and pre-movement testing was used to estimate the surveillance intensity. In both the spatial and spatio-temporal regional models, the fixed effect of the surveillance intensity was not significant for the animal aggregated data, but showed a significant positive posterior mean when farm aggregated data were used 

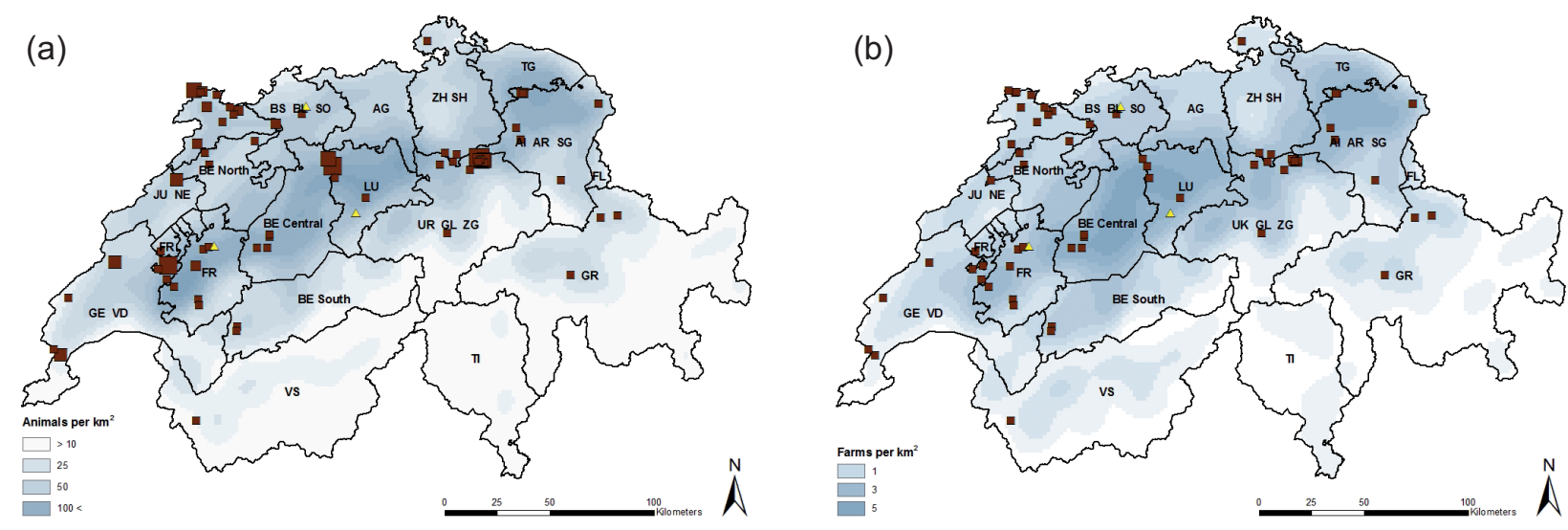

Fig. 2. Maps of Switzerland showing the spatial distribution of BTV-8 infected units recorded in $2008(\square)$ and $2009(\Delta)$. Proportional symbol map of the number of BTV-8 infected cattle, ranging from 1 to 11, at each affected farm (a); and BTV-8 infected farms (b).

Table 1. Summary statistics at animal level and farm level for the 17 bluetongue regions of the population at risk $(n)$, the number of detected positive BTV-8 cases $(y)$ and vaccination coverage (vacc) in Switzerland in 2008 and 2009.

\begin{tabular}{|c|c|c|c|c|c|c|c|c|c|c|c|c|}
\hline \multirow[t]{3}{*}{ BT-region } & \multicolumn{6}{|c|}{ Animals } & \multicolumn{6}{|c|}{ Farms } \\
\hline & \multicolumn{3}{|c|}{2008} & \multicolumn{3}{|c|}{2009} & \multicolumn{3}{|c|}{2008} & \multicolumn{3}{|c|}{2009} \\
\hline & $n$ & $y$ & $\operatorname{Vacc}(\%)$ & $n$ & $y$ & $\operatorname{Vacc}(\%)$ & $n$ & $y$ & $\operatorname{Vacc}(\%)$ & $n$ & $y$ & $\operatorname{Vacc}(\%)$ \\
\hline $\mathrm{FL}$ & 5,683 & 0 & 69 & 6,073 & 0 & 82 & 102 & 0 & 78 & 107 & 0 & 88 \\
\hline $\mathrm{AG}$ & 91,801 & 0 & 72 & 91,913 & 0 & 72 & 2,180 & 0 & 93 & 2,157 & 0 & 92 \\
\hline AI AR SG & 181,773 & 12 & 65 & 183,127 & 0 & 71 & 5,052 & 9 & 88 & 5,042 & 0 & 91 \\
\hline BS BL SO & 74,302 & 1 & 73 & 74,557 & 1 & 77 & 1,828 & 1 & 94 & 1,803 & 1 & 93 \\
\hline FR & 140,714 & 17 & 50 & 142,654 & 1 & 79 & 2,763 & 9 & 79 & 2,754 & 1 & 89 \\
\hline GE VD & 121,871 & 9 & 52 & 123,545 & 0 & 78 & 2,575 & 5 & 79 & 2,551 & 0 & 88 \\
\hline GR & 76,404 & 3 & 37 & 77,998 & 0 & 78 & 2,126 & 3 & 49 & 2,113 & 0 & 87 \\
\hline JU NE & 102,520 & 22 & 68 & 104,757 & 0 & 78 & 1,766 & 11 & 91 & 1,775 & 0 & 92 \\
\hline LU & 152,574 & 17 & 73 & 152,536 & 1 & 78 & 4,492 & 4 & 94 & 4,461 & 1 & 94 \\
\hline TG & 74,962 & 0 & 70 & 75,501 & 0 & 77 & 1,912 & 0 & 89 & 1,891 & 0 & 93 \\
\hline TI & 10,321 & 0 & 29 & 10,512 & 0 & 71 & 455 & 0 & 34 & 444 & 0 & 74 \\
\hline UR GL ZG & 122,130 & 33 & 70 & 121,679 & 0 & 52 & 4,064 & 12 & 88 & 4,035 & 0 & 86 \\
\hline VS & 32,327 & 1 & 32 & 32,826 & 0 & 67 & 1,537 & 1 & 50 & 1,521 & 0 & 82 \\
\hline ZH SH & 110,837 & 4 & 72 & 111,857 & 0 & 71 & 2,686 & 4 & 93 & 2,654 & 0 & 94 \\
\hline BE North & 79,973 & 6 & 59 & 80,593 & 0 & 71 & 2,072 & 4 & 89 & 2,024 & 0 & 88 \\
\hline BE Central & 185,990 & 4 & 64 & 188,854 & 0 & 77 & 6,516 & 4 & 92 & 6,471 & 0 & 94 \\
\hline BE South & 64,253 & 2 & 24 & 63,631 & 0 & 70 & 2,433 & 2 & 40 & 2,399 & 0 & 89 \\
\hline
\end{tabular}

Table 2. The estimated posterior mean and the $95 \%$ posterior credible interval, given as the estimated $2.5 \%$ and $97.5 \%$ quantiles, of the vaccination coverage $\left(\beta_{1}\right)$ and the surveillance intensity $\left(\beta_{2}\right)$ at animal level and farm level of the spatial model (3) and spatio-temporal model (4).

\begin{tabular}{|c|c|c|c|c|c|c|}
\hline & \multicolumn{3}{|c|}{ Spatial model (3) } & \multicolumn{3}{|c|}{ Spatio-temporal model (4) } \\
\hline & $2.5 \%$ & Mean & $97.5 \%$ & $2.5 \%$ & Mean & $97.5 \%$ \\
\hline \multicolumn{7}{|c|}{ (a) Animals } \\
\hline$\beta_{1}$ & -14.59 & -4.30 & 4.61 & -4.97 & -0.25 & 4.80 \\
\hline$\beta_{2}$ & -56.05 & 5.79 & 67.51 & -52.74 & 8.99 & 70.58 \\
\hline \multicolumn{7}{|c|}{ (b) Farm } \\
\hline$\beta_{1}$ & -8.51 & -2.61 & 3.30 & -8.00 & -2.18 & 3.76 \\
\hline$\beta_{2}$ & 12.34 & 32.37 & 52.68 & 16.50 & 37.12 & 58.10 \\
\hline
\end{tabular}


(a)

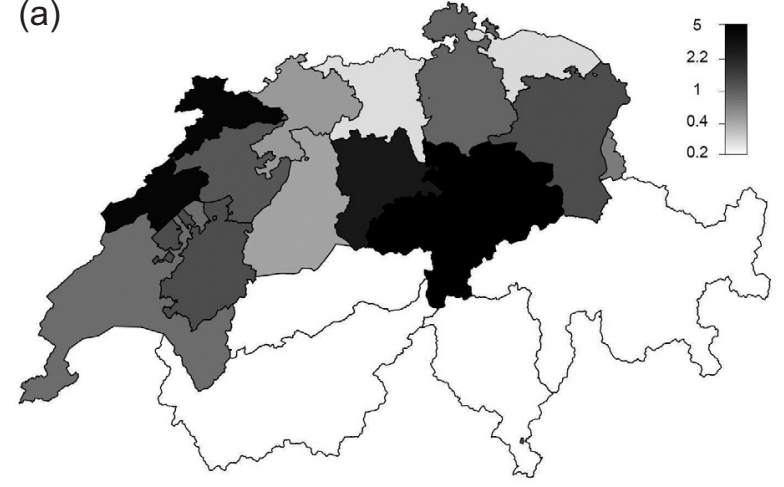

(b)

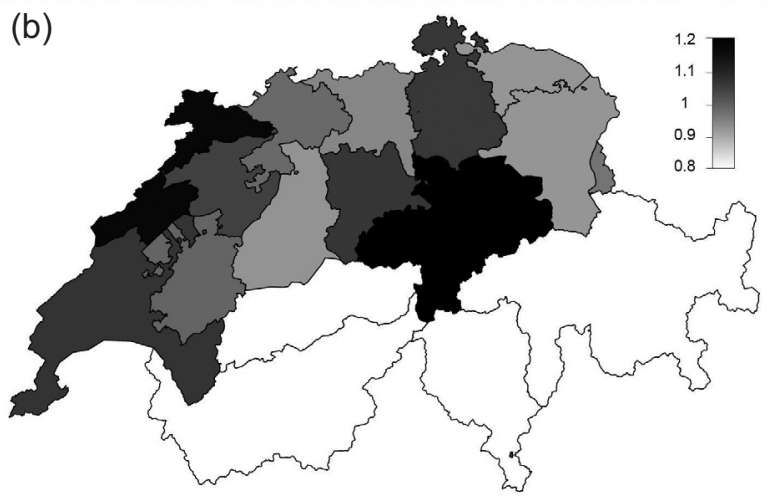

Fig. 3. The estimated relative risk of the spatial model (3) at animal level (a) and farm level (b) after accounting for regional vaccination coverage and surveillance intensity, plotted on an exponential scale $\left(\exp \left(v_{i}+\psi_{i}\right)\right)$. The gray scale bar indicates the relative risk of a unit being BTV-8 infected compared to the overall risk in Switzerland in 2008. Regions in white (GR, TI, VS, BE south) were not considered in the analysis.

(Table 2), supporting that there is a positive association between surveillance intensity and the number of infected farms detected, but a less strong association between surveillance intensity and the number of infected animals detected.

The estimated random intercept for time on a log scale decreased by 3.62 for the animal aggregated data and by 1.71 for the farm aggregated data from 2008 to 2009 , supporting that there was a significant change in BTV-8 prevalence from 2008 to 2009 . However, when the temporal intercept was removed from model (4) (animals: DIC $=86.4$; farms: $\mathrm{DIC}=80.1$ ) to investigate whether the decrease in prevalence of BTV- 8 could be explained by the vaccination coverage alone, the DIC increased (animals: DIC $=212.7$; farms: DIC $=92.5$ ), suggesting that the improved vaccination coverage in 2009 was not sufficient to explain the temporal change in relative risk of a unit being infected.

The estimated relative risk of a unit being infected in 2008 after accounting for regional vaccination coverage and surveillance intensity is presented in Figure 3. The spatial variation between BT-regions was quite large for the models using animal aggregated data, while the range of the residual spatial risk of the farm disease mapping models was narrower, indicating that the models explain most spatial variation in relative disease risk at farm level after adjusting for regional vaccination and surveillance intensity.

\section{Parameter results of geoadditive Bayesian models}

Based on the DIC, the linear spatial trend model (7) $(\mathrm{DIC}=501.0)$ showed a better prediction than model (6) assuming a localized second order random walk $(\mathrm{DIC}=507.6)$, and the latter was, therefore, not considered further.

The estimated effects of the fixed coefficients of model (7) are displayed in Table 3. The posterior means of the longitude $(i)$ and latitude $(j)$ coefficients indicated that the probability of a farm being BTV-8 infected decreased significantly from west to east and increased significantly from south to north. Similarly to the regional analysis, the posterior mean of the vaccination coverage was non-significantly negative and the effect of surveillance intensity was significantly positive.

In a second model (8), the altitude of the farm location was added to the linear spatial trend model to

Table 3. The estimated posterior mean and $95 \%$ posterior credible interval, given as the estimated $2.5 \%$ and $97.5 \%$ quantiles, of the vaccination coverage $\left(\beta_{1}\right)$, surveillance intensity $\left(\beta_{2}\right)$, effect of longitude $\left(\beta_{4}\right)$ and effect of latitude $\left(\beta_{5}\right)$ of the geoadditive linear spatial trend model, without (model 7) and with (model 8) the effect of altitude $\left(\beta_{3}\right)$.

\begin{tabular}{|c|c|c|c|c|c|c|}
\hline & \multicolumn{3}{|c|}{ Geoadditive model (7) } & \multicolumn{3}{|c|}{ Geoadditive model including altitude (8) } \\
\hline & $2.5 \%$ & Mean & $97.5 \%$ & $2.5 \%$ & Mean & $97.5 \%$ \\
\hline$\beta_{1}$ & -0.99 & -0.06 & 1.01 & -1.09 & -0.16 & 0.92 \\
\hline$\beta_{2}$ & 6.38 & 8.26 & 11.08 & 6.42 & 8.31 & 11.13 \\
\hline$\beta_{3}$ & - & - & - & -1.58 & -0.71 & 0.16 \\
\hline$\beta_{4}$ & -0.04 & -0.02 & -0.01 & -0.04 & -0.02 & -0.01 \\
\hline$\beta_{5}$ & 0.004 & 0.03 & 0.06 & -0.01 & 0.02 & 0.05 \\
\hline
\end{tabular}


account for different temperature conditions in the mountain areas. The posterior mean of the altitude was negative, supporting that the probability of a unit being BTV-8 positive decreases with increasing elevation, but the effect was not significant. Furthermore, as is illustrated in Figure 1, the altitude and latitude of Switzerland are highly correlated. As a consequence, the effect of the latitude decreased, indicating that the higher altitude in the Alps accounts for parts of the decreased probability of a farm being BTV-8 infected in the south of Switzerland. The negative effect of the vaccination coverage became slightly stronger when adjusting for the farm altitude, while the effect of the surveillance intensity remained similar to model (7). The DIC was lower when the farm altitude was included as an explanatory variable $(\mathrm{DIC}=500.5)$ but the difference was negligible and, thus, there was no strong preference for either of the models. The residual disease risk after accounting for all explanatory variables decreases from north-west to south-east (Fig. 4). Hence, a trend in the spatial risk surface could still be found after adjustment for the altitude of the farms.

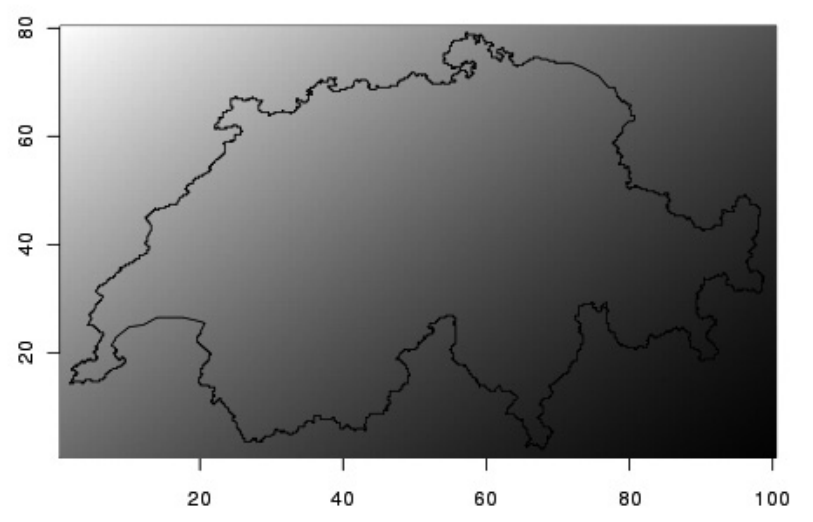

Fig. 4. The estimated relative linear spatial trend obtained from the geoadditive linear spatial trend model (8), adjusted for vaccination coverage, surveillance intensity and altitude. The relative risk ranges from 0.1 (black) to 6.0 (white). The boundary of Switzerland is plotted as an indication of the area under observation.

\section{Discussion}

In this study, the effect of vaccination on the regional and local occurrence of BTV-8 in Switzerland was investigated. Vaccination against BTV-8 was initiated in 2008 , followed by a pronounced decrease in registered BTV-8 cases in 2009. The probability of a unit being infected with BTV-8 was expected to be inversely correlated with vaccination coverage. Although the results of the study point in this direction, a significant effect of vaccination coverage on disease occurrence was not demonstrated.

It may seem contradictory that vaccination is a poor explanatory variable of the probability of a unit being BTV-8 infected. There is an established association between vaccination and the occurrence and spread of infection, and vaccination is an important tool in the control of BT (Caporale et al., 2004; EC, 2009). Nevertheless, in the Alps, there were large areas with a low vaccination coverage as well as a low prevalence of BTV-8. Within the geoadditive model, the effect of vaccination coverage became stronger when the farm altitude was accounted for, suggesting that other factors than solely vaccination influence the probability of a unit being infected with BTV-8. For instance, Szmaragd et al. (2010b) showed that both the vaccination coverage and temperature significantly affect the range of an outbreak.

Additionally, the government prioritised vaccination of areas with a history of BTV-8 outbreaks. Such spatially targeted vaccination could obscure any existing correlation between vaccination coverage and disease prevalence since the vaccination coverage becomes higher in areas with an elevated underlying disease risk. It is also possible that the vaccine uptake increased with proximity to the affected area as presumed infection risk may affect the motivation of animal keepers to vaccinate their livestock (Elbers et al., 2010).

As the analysis was limited to annual time periods, different levels of population susceptibility and disease occurrence throughout the year were not considered. In 2008, the vaccination campaign was not initiated until June and the vaccination of many animals was delayed since they were on summer pasture in the Alps (EC, 2009; FVO, 2009). Cattle receive two vaccine doses 3 to 9 weeks apart, followed by an estimated delay of 3 weeks to reach full protection (Gethmann et al., 2009). Thus, animals considered vaccinated may not have attained full protection against BTV-8 until later in the vector active season. In regions of Italy where mass vaccination against BTV-2 and BTV-9 was not achieved before the start of a new epidemic, vaccination did not significantly reduce disease occurrence, while the spread of infection and disease occurrence decreased significantly in regions with a high vaccination coverage before the start of a new epidemic (Caporale et al., 2004).

Disease outbreaks may appear clustered as a consequence of spatial heterogeneity in surveillance efforts (Kluiters et al., 2008; Birch et al., 2009; Del 
Rio Vilas and Pfeiffer, 2009). To reduce surveillance bias, surveillance intensity was included in the model as an explanatory variable. At farm level, case detection was clearly correlated with surveillance intensity, supporting that spatial heterogeneity in surveillance intensity needs to be considered when assessing spatial occurrence of disease at a national level (Kluiters et al., 2008). The model did not find any significant association between the detection of BTV8 infected cattle and surveillance intensity, possibly due to a lower surveillance intensity at animal level than farm level.

After accounting for vaccination coverage and surveillance intensity, the probability of a farm being infected with BTV-8 increased significantly from east to west and south to north of Switzerland. The observed spatial effect could be related to several ecological factors, such as vector presence and activity, temperature, host density and the disease situation in neighbouring countries. Local environmental conditions and microclimate affect the presence of midges, and the abundance of Culicoides midges varies significantly between different regions of Switzerland (Cagienard et al., 2006; Casati et al., 2009). Due to the environmental conditions, north and northwest of Switzerland is more suitable for Culicoides habitation (Racloz et al., 2007, 2008). The lower relative risk of BTV-8 infection in the south of Switzerland was explained, at least partly, by the higher altitude in the mountain area in the south, and the effect of the latitude became non-significant when the altitude was adjusted for in the model. This agrees with previous studies where lower BTV prevalence was observed at higher elevations in otherwise endemic zones (Ward and Carpenter, 1996) and could be related to a shorter vector active season at higher altitudes (EFSA, 2007) as well as temperature dependent characteristics of vector behaviour and viral replication (Ward and Carpenter, 1996). The northwest of Switzerland is also the most livestock dense area. Simulation studies of BTV spread in Scotland (Szmaragd et al., 2010a) showed that the spatial risk of a holding being BTV-8 infected was associated with livestock and farm densities, where areas of high animal densities allow for onward transmission whereas spread of infection is limited in regions with lower livestock densities.

The maps of residual spatial variation are representations of the relative risk of a unit being BTV-8 infected in a defined area relative to the overall risk in Switzerland (Fig. 3). Nevertheless, Bayesian hierarchical models assume a constant spatial and temporal risk within each region and timeframe. However, the spatial and spatio-temporal divisions may not coincide with changes in disease risk (Pascutto et al., 2000). Since BT is a vector-borne, transboundary disease, BTV-8 prevalence and achieved vaccination coverage in neighbouring countries may also influence the relative disease risk in Switzerland. In 2008, the French vaccination coverage for BTV-8 was low $(36 \%)$, which led to further spread of the disease (EC, 2009) and could contribute to a higher relative disease risk in the west of Switzerland. In Germany, on the other hand, a national vaccination coverage of $85 \%$ was attained with a substantial reduction in disease cases (EC, 2009). Nevertheless, these are national figures and may not be representative for areas bordering Switzerland. In Austrian regions adjacent to Switzerland, the vaccination coverage was also high, and in Italy, BTV-8 infection was secluded to a limited area with a vaccination coverage considered to be satisfactory (EC, 2009), reducing any potential spread of BTV-8 into Switzerland from the east or south.

The role of susceptible species apart from cattle in the maintenance and spread of BTV- 8 was not considered. The level of infection in other susceptible populations and vaccination coverage in sheep and goats could affect the relative risk of BTV-8 infection observed in cattle. For instance, wildlife could act as a potential reservoir for BTV-8 (Szmaragd et al., 2010a). Thus, vaccination data and BTV-8 prevalence in other ruminants should ideally be included in future studies.

\section{Conclusion}

This paper has outlined the application of spatial Bayesian hierarchical models to assess the relative disease risk of BTV-8 at a national level and the effect of vaccination as a method of control of BTV-8. By combining data from different surveillance paths with ecological factors, estimates of relative disease risk were obtained, allowing disease control efforts to be targeted to high risk areas. This is especially a priority in countries where resources available for disease control are limited. Examination of the spatial correlation between disease occurrence, control measures and associated ecological factors is valuable in the evaluation and development of disease control programmes. Especially for diseases with several plausible control strategies, the method could be adapted to evaluate and compare the effect of alternative control measures and serve as a basis in policy development. 


\section{Acknowledgements}

The authors thank the staff at the Swiss Federal Veterinary Office for their support. The study was funded by the Swiss Federal Veterinary Office. RVC manuscript ID number: P/MSc/000108.

\section{References}

Anderson RM, May RM, 1982. Directly transmitted infectious diseases: control by vaccination. Science 215, 1053-1060.

Bernardinelli L, Clayton D, Montomoli C, 1995a. Bayesian estimates of disease maps: how important are priors? Stat Med 14, 2411-2431.

Bernardinelli L, Clayton D, Pascutto C, Montomoli C, Ghislandi M, 1995b. Bayesian analysis of space-time variation in disease risk. Stat Med 14, 2433-2443.

Besag J, Kooperberg C, 1995. On conditional and intrinsic autoregression. Biometrika 82, 733-746.

Besag J, York J, Mollié A, 1991. Bayesian image restoration with two applications in spatial statistics. Ann Inst Stat Math 43, 1-59.

Birch CPD, Chikukwa AC, Hyder K, Del Rio Vilas VJ, 2009. Spatial distribution of the active surveillance of sheep scrapie in Great Britain: an exploratory analysis. BMC Vet Res 5, 23.

Bishop AL, Spohr LJ, Barchia IM, 2004. Effects of altitude, distance and waves of movement on the dispersal in Australia of the arbovirus vector, Culicoides brevitarsis Kieffer (Diptera: Ceratopogonidae). Prev Vet Med 65, 135-145.

Cagienard A, Griot C, Mellor PS, Dension E, Stärk KDC, 2006. Bluetongue vector species of Culicoides in Switzerland. Med Vet Entomol 20, 239-247.

Caporale V, Giovannini A, Patta C, Calistri P, Nannini D, Santucci U, 2004. Vaccination in the control strategy of bluetongue in Italy. Dev Biol (Basel) 119, 113-127.

Casati S, Racloz V, Delécolle JC, Kuhn M, Mathis A, Griot C, Stärk KDC, Vanzetti T, 2009. An investigation on the Culicoides species composition at seven sites in southern Switzerland. Med Vet Entomol 23, 93-98.

Clayton D, Bernardinelli L, 1992. Bayesian methods for mapping disease risk. In: Elliot P, Cuzick J, English D, Stern R (eds.), Geographical and Environmental Epidemiology. Methods for Small Area Studies, Oxford University Press, 205-220 pp.

Clayton DG, Bernardinelli L, Montomoli C, 1993. Spatial correlation in ecological analysis. Int J Epidemiol 22, 1193-1202.

Conraths FJ, Gethmann JM, Staubach C, Mettenleiter TC, Beer M, Hoffmann B, 2009. Epidemiology of bluetongue virus serotype 8, Germany. Emerg Infect Dis 15, 433-435.

Del Rio Vilas VJ, Pfeiffer DU, 2009. The evaluation of bias in scrapie surveillance: a review. Vet J 185, 259-264.

Durr PA, Tait N, Lawson AB, 2005. Bayesian hierarchical mod- elling to enhance the epidemiological value of abattoir surveys for bovine fasciolosis. Prev Vet Med 71, 157-172.

EC, 2009. Emergency vaccination against bluetongue in the EU Nov 2007-Dec 2008, evaluation report. European Commission SANCO/7045/2009.

EFSA, 2007. Bluetongue vectors and vaccines. The EFSA Journal 479, 1-29.

Elbers ARW, Backx A, Meroc E, Gerbier G, Staubach C, Hendrichx G, van der Spek A, Mintiens K, 2008a. Field observations during the the bluetongue serotype 8 epidemic in 2006 I. Detection of first outbreaks and clinical signs in sheep and cattle in Belgium, France and the Netherlands. Prev Vet Med 87, 21-30.

Elbers ARW, Backx A, Mintiens K, Gerbier G, Staubach C, Hendrickx G, van der Spek A, 2008b. Field observations during the bluetongue serotype 8 epidemic in 2006 II. Morbidity and mortality rate, case fatality and clinical recovery in sheep and cattle in the Netherlands. Prev Vet Med 87, 31-40.

Elbers ARW, de Koeijer AA, Scolamacchia F, van Rijn PA, 2010. Questionnaire survey about the motives of commercial livestock farmers and hobby holders to vaccinate their animals against bluetongue virus serotype 8 in 2008-2009 in the Netherlands. Vaccine 28, 2473-2481.

FVO, 2009. Blauzungenkrankheit in der Schweiz. Federal Veterinary Office. Available at: <http://www.bvet.admin. ch/gesundheit_tiere/01973/02437/index.html?lang=de\&down load=NHzLpZeg7t, lnp6I0NTU04212Z6ln1acy4Zn4Z2qZpn O2Yuq2Z6gpJCEeYJ_fWym162epYbg2c_JjKbNoKSn6A-; accessed 5 May 2010.

Gethmann J, Hüttner K, Heyne H, Probst C, Ziller M, Beer M, Hoffmann B, Mettenleiter TC, Conraths FJ, 2009. Comparative safety study of three inactivated BTV-8 vaccines in sheep and cattle under field conditions. Vaccine 27, 41184126.

Gschössl S, Czado C, 2008. Modelling count data with overdispersion and spatial effects. Stat Pap 49, 531-552.

Hofmann MA, Griot C, Chaignat V, Perler L, Thür B, 2008. Bluetongue disease reaches Switzerland. Schweiz Arch Tierheilkd 150, 49-56.

Hofmann MA, Renzullo S, Mader M, Chaignat V, Worwa G, Thuer B, 2008. Genetic characterization of Toggenburg orbivirus, a new bluetongue virus, from goats, Switzerland. Emerg Infect Dis 14, 1855-1861.

Kluiters G, Chaignat V, Schwermer H, 2008. Spatial distribution of bluetongue surveillance and cases in Switzerland. Schweiz Arch Tierheilkd 150, 543-522.

Kneib T, Fahrmeir L, 2006. Structured additive regression for categorical space-time data: a mixed model approach. Biometrics 62, 109-118.

Knorr-Held L, 2000. Bayesian modelling of inseperable spacetime variation in disease risk. Stat Med 19, 2555-2567.

Mellor PS, 2000. Replication of arboviruses in insect vectors. J 
Comp Pathol 123, 231-247.

Mellor PS, Boorman J, Baylis M, 2000. Culicoides biting midges: their role as arbovirus vectors. Annu Rev Entomol 45, 307-340.

Mellor PS, Wittmann EJ, 2002. Bluetongue virus in the Mediterranean basin 1998-2001. Vet J 164, 20-37.

Méroc E, Faes C, Herr C, Staubach C, Verheyden B, Vanbinst T, Vandenbussche F, Hooyberghs J, Aerts M, De Clercq K, Mintiens K, 2008. Establishing the spread of bluetongue virus at the end of the 2006 epidemic in Belgium. Vet Microbiol 131, 133-144.

Mintiens K, Méroc E, Mellor PS, Staubach C, Gerbier G, Elbers ARW, Hendrickx G, De Clercq K, 2008. Possible routes of introduction of bluetongue virus serotype 8 into the epicentre of the 2006 epidemic in north-western Europe. Prev Vet Med 87, 131-144.

Musio M, Augustin H, von Wilpert K, 2008. Geoadditive Bayesian models for forestry defoliation data: a case study. Environmetrics 19, 630-642.

Pascutto C, Wakefield JC, Best NG, Richardson S, Bernardinelli L, Staines A, Elliott P, 2000. Statistical issues in the analysis of disease mapping data. Stat Med 19, 2493-2519.

Purse BV, Mellor PS, Rogers DJ, Samuel AR, Mertens PPC, Baylis M, 2005. Climate change and the recent emergence of bluetongue in Europe. Nat Rev Microbiol 3, 171-181.

R Development Core Team, 2010. R: a language and environment for statistical computing. R Foundation for Statistical Computing. Available at: http://www.R-project.org (accessed 9 June 2010).

Racloz V, Presi P, Vounatsou P, Schwermer H, Casati S, Vanzetti T, Griot C, Stärk KDC, 2007. Use of mapping and statistical modelling for the prediction of bluetongue occurrence in Switzerland based on vector biology. Vet Ital 43, 513-518.

Racloz V, Venter G, Griot C, Stärk KDC, 2008. Estimating the temporal and spatial risk of bluetongue related to the incursion of infected vectors into Switzerland. BMC Vet Res 4, 42 .
Roy P, Boyce M, Noad R, 2009. Prospects for improved bluetongue vaccines. Nat Rev Microbiol 7, 120-128.

Rue H, Held L, 2005. Gaussian Markov random fields, Chapman and Hall, 101-117 pp.

Rue H, Martino S, Chopin N, 2009. Approximate Bayesian inference for latent Gaussian models by using integrated nested Laplace approximations. J R Stat Soc Series B Stat Methodol 71, 319-392.

Rüsch P, Kihm U, 2003. The federal system of veterinary services in Switzerland. Rev Sci Tech 22, 423-432.

Saegerman C, Berkvens D, Mellor PS, 2008. Bluetongue epidemiology in the European Union. Emerg Infect Dis 14, 539-544.

Schrödle B, Held, L, 2010. A primer on disease mapping and ecological regression using INLA. Comput Stat 26, 241-258.

Sellers RF, 1984. Bluetongue in Africa, the Mediterranean region and Near East - disease, virus and vectors. Prev Vet Med 2, 371-378.

Spiegelhalter DJ, 2002. Bayesian measures of model complexity and fit. J R Stat Soc Series B Stat Methodol 64, 583-639.

Szmaragd C, Gunn GH, Gubbins S, 2010a. Assessing the consequences of an incursion of a vector-borne disease. II. Spread of bluetongue in Scotland and impact of vaccination. Epidemics 2, 139-147.

Szmaragd C, Wilson AJ, Carpenter S, Wood JLN, Mellor PS, Gubbins S, 2010b. The spread of bluetongue virus serotype 8 in Great Britain and its control by vaccination. PLoS One 5, e9353. Vellema P, 2008. Bluetongue in sheep: question marks on bluetongue virus serotype 8 in Europe. Small Rumin Res 76, 141148.

Ward MP, Carpenter TE, 1996. Simulation modeling of the effect of climatic factors on bluetongue virus infection in Australian cattle herds. I. Model formulation, verification and validation. Prev Vet Med 27, 1-12.

Wittmann EJ, Mellor PS, Baylis M, 2002. Effect of temperature on the transmission of orbiviruses by the biting midge, Culicoides sonorensis. Med Vet Entomol 16, 147-156. 